\title{
DETERMINATION OF AVALANCHE DYNAMICS FRICTION COEFFICIENTS FROM MEASURED SPEEDS
}

\author{
by \\ D. M. McClung and P. A. Schaerer \\ (Division of Building Research, National Research Council Canada, Vancouver, \\ British Columbia V6R 1P5, Canada)
}

\begin{abstract}
An avalanche dynamics model, appropriate for complex terrain, for real avalanche paths was developed by Perla, Cheng and McClung in 1980. The model has two friction terms, one for sliding friction which is independent of speed, and one for turbulent friction which is proportional to $\mathrm{V}^{2}$, where $\mathrm{V}$ is the centreof-inass speed along the incline. By introducing speed maxima for avalanches, along with start and stop reference positions, it is possible to determine the the two constant friction coefficients for the model. When this is done, it is found that speed data often exceed a model spced limit implied by the application of $V=0$ at the start and stop positions. This effect is illustrated by analytic solutions of the relevant equations, as well as numerical solutions for actual avalanche paths. Some limitations and properties of the fundamental modelling are outlined and suggestions given for future use of such models.
\end{abstract}

\section{INTRODUCTION}

Dynamics models are used when the speed of avalanches must be estimated for calculations of impact pressures, and when the runout distances of avalanches are required. Such applications require a knowledge of the friction coefficients in the drag terms of the models.

Centre-of-mass avalanche dynamics models describe the speed $V$ of the centre-of-mass of the avalanche along the incline. The first such model, introduced by Voellmy (1955), was extended by Körner (1976) and Perla and others (1980). The latter, referred to here as the PCI (Perla, Cheng, McClung) model, is a particularly important extension. It enables one to follow the progress of the avalanche over geometrically complex paths between defined reference points (start and stop positions) rather than over a simplistic two-segment avalanche path with its need to estimate the mid-path point of the beginning of deceleration, as prescribed by Voellmy. Other potential extensions introduced by PCI include the possibility of handling snow entrainment, centripetal forces, and momentum corrections for abrupt changes in terrain, al though these extensions are mainly in advance of any known data on these effects.

The actual drag terms for the Voelliny and PCM models are mathematically identical and coupled similarly so that, al though the PCM model is used in the present paper, the general conclusions will apply to Voellmy's model and Körner's work also. Unfortunately, it is not possible to determine values of the friction coefficients for these centre-of-mass models without speed data. When avalanche speed maxima are introduced, however, along with measured avalanche start and stop positions, the solution over the terrain profile gives the friction coefficients uniquely for the PCM model. This not only provides friction coefficients for actual avalanches, but illustrates some important properties of the basic modelling that must be understood before applications are attempter.

\section{THEORETICAL CONSIDERATIONS}

The PCM model describes the motion of the centreof-mass of an avalanche along the avalanche path from start to stop positions. The model contains two friction terms: (1) a speed-independent term which represents the average value $\bar{\mu}$ of sliding friction over the length of the path $S_{0}$ from start to stop positions, and (2) a term proportional to $V^{2}$ representing the average value of the turbulent drag over the path length. This term is defined by a coefficient written as a constant ratio of drag $\bar{D}$ to mass $\bar{M}$, or $\bar{D} / M$.

The differential equation governing the motion of the centre-of-mass for the PCM model is:

$$
\frac{d V^{2}}{d S}=2 g[\sin \theta(S)-\bar{\mu} \cos \theta(S)]-2 \bar{D} V^{2} / M,
$$

where dS is an element of path length, $g$ is acceleration due to gravity, and $\Theta(S)$ is the local slope angle (Fig.1). Since path radius-of-curvature effects are proportional to $\mathrm{V}^{2}$, these may be included in the overall specification of $\bar{D} / M$. From Equation (1) it is clear that $\bar{\mu}$ is dimensionless whereas $\bar{D} / M$ is proportional to an analogous turbulent drag coefficient but has dimensions of (1 ength) $)^{-1}$.

$$
\begin{aligned}
& \text { The general solution to Equation (1) is: } \\
& \begin{aligned}
V(S) & =\left\{\operatorname { e x p } ( - 2 \overline { D } S / M ) \left[C_{0}+\int 2 g[\sin \Theta(S)-\right.\right. \\
& -\mu \overline{c o s} \Theta(S)] \exp (2 D \bar{S} / M) d S]\}^{1 / 2}
\end{aligned}
\end{aligned}
$$

where $C_{0}$ is a constant determined by initial conditions.

To illustrate the properties of the model in relation to some effects found with speed data on geometrically complex avalanche paths, Equation (2) is solved here along the arc of a circular path. The 


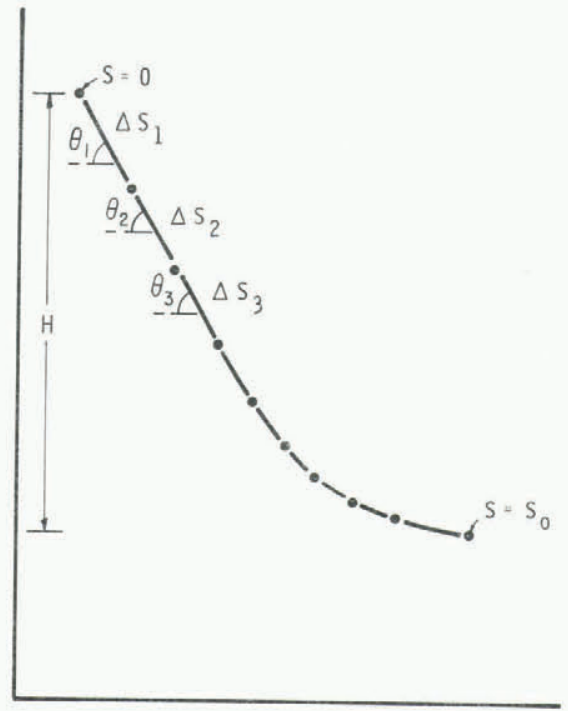

Fig.1. Schematic of avalanche path broken into segments. The segments are of length $\Delta S_{j}$ with a total path length $S_{0}=\Sigma_{i} \Delta S_{j}$ and the total vertical drop is denoted by $H$. Each segment $(i)$ is specified by its length and slope angle $\Theta$.

circular path has the advantage that the analytic solution is of simple form. Kozik (1962) also studied this problen. 0 ther profiles, such as the parabola, are perhaps better theoretical models of actual avalanche terrain, but no simple analytic solutions are available.

Consider the circular path $\theta(S)=\theta_{0}\left(1-S / S_{0}\right)$ where $\theta_{0}$ is the initial or starting zone angle, and $S_{0}$ is the total length of arc along the incline until the valley floor is reached where $\theta\left(S_{0}\right)=0$. With $V(0)=0$, the solution of Equation (2) is then:

$V(S)=C\left\{A\left[\sin \theta_{0}\left(1-S / S_{0}\right)-\sin \theta_{0} \exp (-2 \bar{D} S / M)\right]+\right.$

$+B\left[\cos \theta_{0}\left(1-S / S_{0}\right)-\cos \theta_{0} \exp (-2 \bar{D} S / M)\right\}^{1 / 2}$

where

$$
C=\frac{\left[2 g\left(S_{0} / \theta_{0}\right)\right]^{1 / 2}}{\left[\left(2 \bar{D} S_{0} / M \theta_{0}\right)^{2}+1\right]^{1 / 2}}, \quad B=1-\left(2 \mu D S_{0} / M \theta_{0}\right)
$$

and

$$
\mathrm{A}=2 \overline{\mathrm{DS}} \mathrm{S}_{0} / \mathrm{M}_{0}+\bar{\mu} .
$$

Now consider stop positions on the incline. Application of the boundary condition $\mathrm{V}\left(\mathrm{KS}_{0}\right)=0$ where $0<K<1$ gives from Equation ( 3 ),

$$
\bar{\mu}=\frac{1-2 \bar{D} S_{0} / M \theta_{0} f(\bar{D} / M)}{2 \bar{D} S_{0} / M \theta_{0}+f(\bar{D} / M)}
$$

where

$$
f(\bar{D} / M)=-\frac{\sin \theta_{0}-\sin \left[\theta_{0}(1-K)\right] \exp \left(2 \overline{D K S_{0}} / M\right)}{\cos \theta_{0}-\cos \left[\theta_{0}(1-K)\right] \exp \left(2 \overline{D K} S_{0} / M\right)}
$$

Equation (4) gives a relationship between $\bar{\mu}, \bar{D} / \mathrm{M}$ and path parameters where each pair $\frac{\mu}{\mu}, \bar{D} / M$ implies a maximum speed. Figure 2 depicts such a relationship for $K=1, \theta_{0}=40^{\circ}$ and $\bar{D} / M$ scaled inversely proportional to total path length $S_{0}$. Körner (1981) and Bakkehøi and others (1981) deterimined similar relationships for actual avalanche paths.

This analysis indicates that to determine $\bar{\mu}$ and $\bar{D} / M$ for a given avalanche, an estimate of maximum speed or a similar estimate of speed somewhere along

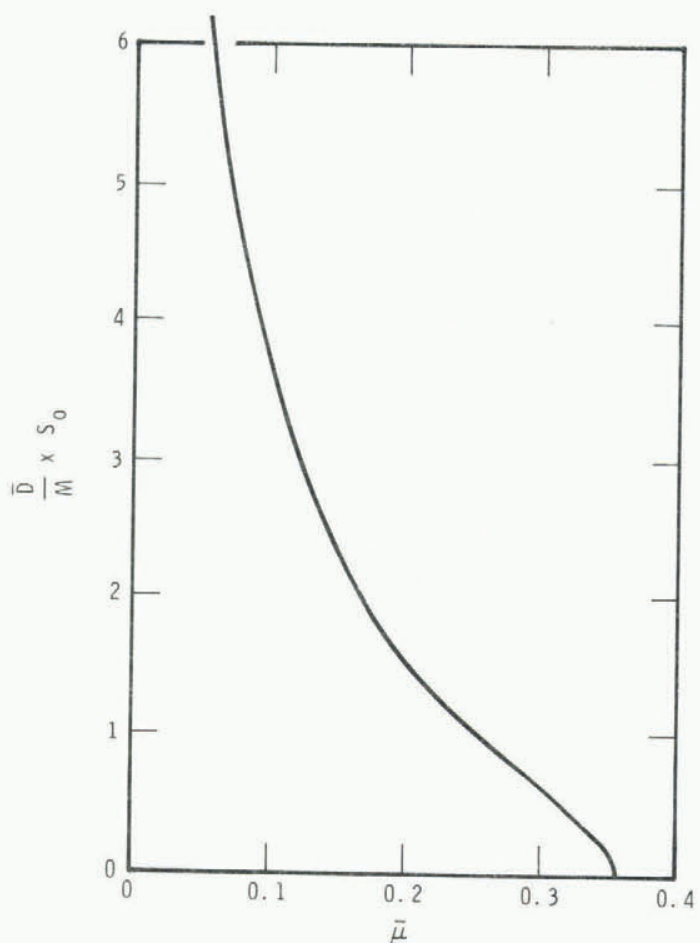

Fig.2. The relation between $\bar{\mu}$ and $\bar{D} / M$ implied by the conditions $V(0)=V\left(S_{0}\right)=0$ for a circular path with $\theta_{0}=40^{\circ}$. Maximum speed increases as $\vec{\mu}$ increases and $\bar{D} / M$ decreases. The values as $\bar{D} / M \rightarrow 0$ correspond to the high speed limit where $V_{M}=V_{M}^{\prime}$.

the incline is needed. Otherwise no values can be attached to $\bar{\mu}$ and $\bar{D} / M$ because an infinite number of pairs is generated by application of the boundary conditions $V=0$ at the start and stop positions.

An added result from Equation (4) is that when $\bar{D} / M<<1 / 2 K S_{0}$ and for $0<K<1$ and $\theta_{0}$ in the range typical for avalanche paths, $\bar{\mu}$ approaches a maximum value given by $\bar{\mu}_{\max }=\tan \left[\theta_{0}(2-K) / 2\right]$ where the argument $\left[\theta_{0}(2-k) / 2\right]$ represents the average angle along the incline between $S=0$ and the stop position $S=K S_{0}$. Also, sensitivity to $\bar{D} / M$ is lost in the equations and the expression for maximum speed $V_{M}$ becomes an upper limit value or model speed limit $V_{i}$, which occurs at $S=K S_{0} / 2$ and is given by:

$$
\begin{aligned}
V_{\bar{M}} / \sqrt{S_{0}}= & {\left[2 g / \Theta_{0}\right]^{1 / 2}\left\{\bar{\mu}_{\max }\left[\sin \Theta_{0}(1-K / 2)-\sin \theta_{0}\right]+\right.} \\
& \left.+\cos \theta_{0}(1-K / 2)-\cos \Theta_{0}\right\}^{1 / 2} .
\end{aligned}
$$

For example, if $K=1$, then for the usual range of avalanche starting-zone angles, $30^{\circ} \leqslant \theta_{0} \leqslant 65^{\circ}$, the speed limit is in the range $1.15 \leqslant V_{M} / \sqrt{S_{0}} \leqslant 1.80$. Similarly, V. drop, $H$, since for these profiles $S_{Q}=1.45 \mathrm{H}$ for $\theta_{0}=65^{\circ}$ and $S_{0}=3.27 \mathrm{H}$ for $\theta_{0}=30^{\circ}$ so that $V_{\bar{M}} / \sqrt{H}=2.16$ for $\theta_{0}=65^{\circ}$ and $V_{M} / \sqrt{H}=2.08$ for
$\theta_{0}=30^{\circ}$.

Given $V(0)=V\left(K S_{0}\right)=0$ and $\bar{\mu}, \bar{D} / M$ both constant, $\bar{\mu}$ must be increased as $\bar{D} / M$ is decreased as Equation (4) and Figure 2 depict. The model speed limit corresponds to the case where sensitivity to the $\mathrm{V}^{2}$ or turbulent drag is lost in the equations and the avalanche must have all of its drag as sliding friction. This is not physically realistic and $\bar{\mu}$ is forced toward higher values as the maximum speed increases toward V

Another result from Equation (4) is that given a value of $\bar{D} / M$, then $\bar{\mu}$ is very sensitive to the slope angle $\theta_{f}$ where the avalanche stops. For example, if

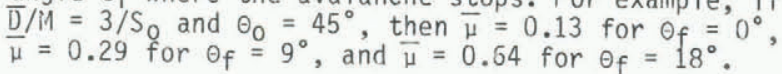


TABLE I. ILLUSTRATION OF THE DECREASE IN VM AND INCREASE IN $\bar{\mu}_{\max }$ WITH INCREASING STOPPING ANGLE FOR A CIRCULAR PATH WITH $\theta_{0}=45^{\circ}$

$\begin{array}{lrccc}K & \theta_{f} & V_{\tilde{M}} / \sqrt{S_{0}} & V_{\tilde{\mu}} / \sqrt{H} & \bar{\mu}_{\max } \\ 1.0 & 0^{\circ} & 1.45 & 2.38 & 0.41 \\ 0.8 & 9^{\circ} & 1.17 & 1.77 & 0.51 \\ 0.4 & 18^{\circ} & 0.61 & 0.87 & 0.73\end{array}$

The model speed limits $V$ G are also sensitive to the stop position. Table I gives examples of the model limits as a function of the stop position angle. This table shows that $V_{\mathscr{G}} / \sqrt{S_{0}}$ and $V_{\mathscr{V}} / \sqrt{H}$ decline rapidly as the stop angle increases. This is important as many avalanches for which speed measurements were available stopped on relatively steep slopes, and the model speed limits are exceeded in a number of cases by this effect.

\section{SPEED MEASUREMENTS OF AVALANCHES}

During the winters between 1966 and 1972, avalanche speeds and runout distances were measured on paths with known profiles at Rogers Pass, British Columbia, Canada. The speed measurements were described by Schaerer (1975) and they represent near maximum speed in the middle portion of the path. The speed data, together with start and stop positions at the tip of the debris and the profile of the entire avalanche path, are sufficient to determine the model friction coefficients of the observed avalanches.

In the model the speeds represent centre-of-mass speeds whereas the observations are actually frontal speeds. It is assumed, however, that the frontal speed is a good approximation of the centre-of-mass speed. Salway (1978) provided data from Rogers Pass which indicate that this is true in some instances, and later measurements there confirm his conclusions.

The stop position for the model corresponds to that for the centre-of-inass, whereas the field data correspond to the rest position of the tip of the avalanche. This, with the previous theoretical analysis, means that the actual $\bar{\mu}$ values obtained will normally be less than the actual values for centre-of-mass stop positions as the centre-of-mass of the actual avalanche is usually on a steeper slope. Also, this procedure will produce values of $\bar{D} / M$ slightly higher than those for actual avalanche centre-of-mass positions. These considerations actually enhance the major conclusions of this paper, as shown later.

\section{DETERMINATION OF FRICTION COEFFICIENTS}

Since the avalanche paths are complex, the velocity profiles have been solved numerically by breaking the paths into segments of length $\Delta S_{j}$ and slope angles $\theta_{i}$ (Fig.1), as described by PCM. Using this procedure, the solution that best matches the start position, the stop position within $\pm 5 \mathrm{~m}$, and the speed weasurement at its position along the profile, was found by a trial and error iteration procedure to give the values of $\bar{\mu}$ and $\bar{D} / M$. Normally, about ten computer runs were needed to match these data and yield the values of the friction coefficients for each avalanche.

Table II gives the friction coefficients $\bar{\mu}$ and $\bar{D} / M$, determined by the procedure outlined above. Some points of note from Table II are: (1) there are a number of events $(8,25,26,27,30)$ in which the measured speed exceeds the model speed limit, and another group for which the solutions are essentially insensitive to variations in $\bar{D} / M(5,18,34)$, (2) wany of the values of $\bar{\mu}$ are very high and they often approach expected static values of sliding friction. Given that slab avalanches start on slopes as low as $25^{\circ}$, static values near 0.5 are implied. In the model, $\bar{\mu}$ should depend on the properties of flowing snow and should represent an average value over the
TABLE II. FRICTION PARAMETERS FOR AVALANCHES

\begin{tabular}{|c|c|c|c|c|}
\hline Avalanche & $\begin{array}{l}\text { Mid-path } \\
\text { speed } \\
\text { estimate } \\
\left(m \mathrm{~s}^{-1}\right)\end{array}$ & $\bar{\mu}$ & $1000 \bar{D} / \mathrm{M}$ & $\begin{array}{l}\text { Slope angle } \\
\text { of stop } \\
\text { position } \\
\left({ }^{\circ}\right)\end{array}$ \\
\hline 1. Stone Arch & 28 & 0.36 & 4.2 & 17 \\
\hline 2. Stone Arch & 43 & 0.44 & 1.6 & 17 \\
\hline 3. Portal & 18 & 0.68 & 5.1 & 34 \\
\hline 4. Portal & 12 & 0.47 & 11.0 & 16 \\
\hline 5. Portal & 22 & $\star \star \star$ & & 28 \\
\hline 6. Portal & 21 & 0.68 & 3.2 & 28 \\
\hline $\begin{array}{l}\text { 7. McDonald-5 } \\
\text { 8. Tupper Cliff }\end{array}$ & 24 & 0.47 & 4.0 & 19 \\
\hline 8. Tupper Cliff & 18 & * & * & 26 \\
\hline 9. Tupper Minor & 12 & 0.32 & 29.0 & 0 \\
\hline 10. Tupper Minor & 14 & 0.02 & 31.0 & 0 \\
\hline 11. Len's East & 17 & 0.46 & 11.0 & 18 \\
\hline 12. Len's West & 42 & 0.42 & 1.5 & 18 \\
\hline 13. Len's West & 16 & 0.36 & 11.0 & 18 \\
\hline 14. Double Bench & 23 & 0.62 & 3.0 & 21 \\
\hline 15. Double Bench & 35 & 0.50 & 2.0 & 21 \\
\hline 16. Double Bench & 22 & 0.43 & 3.3 & 21 \\
\hline 17. Single Bench & 19 & 0.54 & 3.7 & 20 \\
\hline 18. Single Bench & 40 & $\star \star$ & 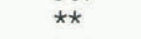 & 20 \\
\hline 19. Single Bench & 23 & 0.46 & 3.1 & 20 \\
\hline 20. Single Bench & 27 & 0.64 & 0.5 & 22 \\
\hline 21. Single Bench & 12 & 0.40 & 12.0 & 20 \\
\hline 22. Single Bench & 17 & 0.54 & 3.3 & 28 \\
\hline 23. Single Bench & 26 & 0.54 & 3.8 & 28 \\
\hline 24. Single Bench & 25 & 0.37 & 5.4 & 20 \\
\hline 25. Single Bench & 50 & * & $\star$ & 20 \\
\hline 26. Mounds & 35 & * & * & 21 \\
\hline 27. Crossover & 62 & * & * & 26 \\
\hline 28. Lone Pine & 47 & 0.34 & 0.97 & 13 \\
\hline 29. Lone Pine & 41 & 0.29 & 1.7 & 13 \\
\hline 30. Lone Pine & 34 & * & * & 28 \\
\hline $\begin{array}{l}\text { 31. McDonald } \\
\text { West-4 }\end{array}$ & 38 & 0.17 & 2.6 & 0 \\
\hline 32. McDonald & & & & \\
\hline $\begin{array}{l}\text { West-4 } \\
\text { 33. McDonald West }\end{array}$ & 19 & 0.10 & 13.0 & 0 \\
\hline $\begin{array}{l}\text { 33. McDonald West } \\
\text { Shoulder }\end{array}$ & 25 & 0.05 & 7.7 & 0 \\
\hline 34. McDonald West & & $\star \star \star$ & & \\
\hline $\begin{array}{l}\text { Shoulder } \\
\text { 35. Gunner's }\end{array}$ & $\begin{array}{l}29 \\
23\end{array}$ & 0.46 & $\begin{array}{l}* * \\
5.0\end{array}$ & 13 \\
\hline $\begin{array}{l}\text { 35. Gunner's } \\
\text { 36. Gunner's }\end{array}$ & $\begin{array}{l}23 \\
41\end{array}$ & $\begin{array}{l}0.40 \\
0.55\end{array}$ & $\begin{array}{l}.0 \\
1.0\end{array}$ & 17 \\
\hline $\begin{array}{l}\text { 36. Gunner's } \\
\text { 37. Gunner's }\end{array}$ & $\begin{array}{l}41 \\
32\end{array}$ & 0.33 & 3.6 & 17 \\
\hline 38. Gunner's & 41 & 0.33 & 2.2 & 17 \\
\hline
\end{tabular}

entire path. The data in Table II, however, as well as the analytic solutions show that the constraints introduced make $\bar{\mu}$ strongly dependent on the stopping angle as well as path characteristics.

\section{DISCUSSION}

The results in Table II can be interpreted, along with the analytic solutions obtained previously, in terms of some conclusions. (1) A number of avalanches, for which measured speeds are presented, were observed to stop on steep slopes with angles higher than $10^{\circ}$. With the model, the speed limit declines rapidly with increasing stopping angle whereas, in actual avalanches, it apparently does not. (2) Specification of the boundary conditions $V=0$ at the path end points, implies a relationship between $\frac{t}{\mu}$ and $\bar{D} / M$, e.g. Equation (4) (for the circular path) which indicates that as $\bar{D} / M$ is decreased $\bar{\mu}$ must be increased in order to match the stop position for higher values of maximum speed. Expressions such as this are no doubt unrealistic at the high speed linit when $\bar{D} / M<<1 / 2 S_{0}$ and many of the data approach or exceed this high speed limit. Relationships like Equation

\footnotetext{
* Cases for which measured speed exceeds model limits.

** Cases for which solution is indeterminate and insensitive to variations in $\bar{D} / M$.
} 
(4) imply that $\bar{\mu}$ increases with increasing maximum speed, which is contrary to the views of some researchers. Voellmy (1955) and Schaerer (1975) imply that $\mu$ decreases rather than increases with speed.

Without further data and theoretical work it is difficult to resolve this question but it is possible that expressions such as shown in Equation (4) are physically unrealistic in general, not just at the high speed limit. If so, it is essential that speed data be used to determine the coefficients in the model because application of start and stop boundary conditions alone will generate an infinite number of pairs of $\bar{\mu}$ and $\bar{D} / M$, of which one pair at most may have meaning. Simply stated, the differential equation implies that $\bar{\mu}$ and $\bar{D} / M$ are average values for the entire path dependent on the properties of flowing snow, whereas application of $V=0$ at the path end points, implies that these drag coefficients are coupled in a specific way and depend sensitively on stopping angle and other terrain parameters. This procedure can produce coefficients different than might be expected from properties of flowing snow.

There are several options available, at present, to deal with these problems. If the restriction of constant $\bar{\mu}$ and $\bar{D} / M$ is relaxed, it may well be possible to explain the measured field data and produce physically realistic parameter relationships. For example, if $\mu$ and $D / M$ were to decrease and increase together as speed increases and decreases then local values of high speed would be possible with perhaps enough friction to stop the avalanche on steep slopes once deceleration begins. This approach, however, complicates the problem. Although it may seem easy to postulate constitutive equations between $\mu$ and $D / M$ as functions of speed or path parameters, at present there are not enough data, nor has theoretical work advanced far enough, to know the form of such equations.

Similarly, introduction of a more complex model, such as inclusion of a drag term linear in $\mathrm{V}$ to account for viscous drag (Salm 1966), would give a more flexible algorithm. Such a formulation, however, would make it impossible to determine the parameters, uniquely in the model, from existing avalanche speed data and the characteristics of flowing snow. As PCM have indicated, even if the full velocity profile were known all along the incline, it would not be possible to determine the parameters uniquely for models with more than two constant parameters if velocity boundary conditions are adhered to.

Collectively the data, calculations and theory point to some inconsistencies regarding properties of the model when $\mu$ and $D / M$ are assuned constant, and what is expected and what actually occurs. If friction is assumed constant over the path, as available data demand, there are at least two options available. One of these was outlined by PCI in terms of a one-parameter model. Another is that with a knowledge of the properties of the two-parameter model and an understanding of actual behaviour, it may still be possible to apply the model for restricted classes of problems in a way that will avoid the pitfalls and inconsistencies. That is, by keeping the properties of the model clearly in mind and separate from the expected properties of flowing snow, it may be possible to use the model judiciously and provide engineering estimates of run-out for certain eventualities. This latter option appears to be a promising approach and will be reported in a future publication. Clearly, this class of two-parameter models cannot be used for all types of avalanches, terrain, and stopping angles as normally applied. The basic simplicity of the models dictates that there are limitations on their validity.

\section{ACKNOWLEDGEMENTS}

Many of the ideas presented are the results of discussions with R Perla, Environment Canada. This paper is a contribution from the Division of Building Research, National Research Council of Canada, and is published with the approval of the Director of the Division.

\section{REFERENCES}

Bakkehøi S, Cheng T, Domaas U, Lied K, Perla R, Schieldrop B 1981 On the computation of parameters that model snow avalanche motion. Canadian Geotechnical Journal 18(1): 121-130

Körner H J 1976 Reichweite und Geschwindigkeit von Bergstürzen und Fliesschneelawinen. Rock Mechanics $8(4)$ : 225-256

Körner H J 1981 Modelle zur Berechnung der Bergsturz- und Lawinenbewegung. Interpraevent 1980 Band 2: $15-55$

Kozik S M 1962 Raschet dvizheniya snezhnykh lavin [Calculation of snow avalanches]. Leningrad, Gidrometeoizdat

Perla R, Cheng T T, McClung D M 1980 A two-parameter model of snow-avalanche motion. Journal of Glaciology 26(94): 197-207

Salm B 1966 Contribution to avalanche dynamics. Intermational Association of Scientific Hydrology Publication 69 (Symposium of Davos 1965 - Scientific Aspects of Snow and Ice Avalanches): 199-214

Salway A A 1978 A seismic and pressure transducer system for monitoring velocities and impact pressures of snow avalanches. Arctic and Alpine Research 10(4): 769-774

Schaerer P A 1975 Friction coefficients and speed of flowing avalanches. Intemational Association of Hydrological Sciences Publication 114 (Symposium of Grindelwald 1974 - Snow Mechanics): 425-432

Voellmy A 1955 Uber die Zerstörungskraft von Lawinen. Schweizerische Bauzeitung 73(12): 159-162, 73(15): $212-217,73(17): 246-249,73(19): 280-285$ 